\title{
Low frequency electromagnetic fluctuations in Kappa magnetized plasmas
}

\author{
Sunjung Kim ${ }^{1}$, M. Lazar ${ }^{1,2}$, R. Schlickeiser ${ }^{1}$ R. A. López ${ }^{3}$, P. H. \\ Yoon $^{3,4,5}$ \\ ${ }^{1}$ Institut für Theoretische Physik, Lehrstuhl IV: Weltraum- und Astrophysik, \\ Ruhr-Universität Bochum, D-44780 Bochum, Germany \\ ${ }^{2}$ Centre for Mathematical Plasma Astrophysics (CmPA), University of Leuven, \\ Celestijnenlaan 200B, 3001 Leuven, Belgium \\ ${ }^{3}$ Institute for Physical Science and Technology, University of Maryland, College Park, \\ Maryland 20742, USA \\ ${ }^{4}$ School of Space Research, Kyung Hee University, Yongin, Gyeonggi 446-701, Korea \\ ${ }^{5}$ Korea Astronomy and Space Science Institute, Daejeon 305-348, Korea
}

\begin{abstract}
The present paper provides a theoretical approach for the evaluation of the low-frequency spontaneously emitted electromagnetic fluctuations in Kappa magnetized plasmas, which include the kinetic Alfvén, fast magnetosonic/whistler, kinetic slow mode, ion Bernstein cyclotron modes, and higher-order modes. The model predictions are consistent with particle-in-cell simulations. Effects of suprathermal particles on low-frequency fluctuations are studied by varying the power index, either for ions $\left(\kappa_{i}\right)$ or for electrons $\left(\kappa_{e}\right)$. Computations for an arbitrary wave vector orientation and wave polarization provide the intensity of spontaneous emissions to be enhanced in the presence of suprathermal populations. These results strongly suggest that spontaneous fluctuations may significantly contribute to the electromagnetic fluctuations observed in space plasmas, where suprathermal Kappadistributed particles are ubiquitous.
\end{abstract}

PACS numbers: 52.65.Rr, 52.35.Hr, 52.35.Ra, 52.25.Os 


\section{INTRODUCTION}

A permanent low frequency turbulence exists in the solar wind [1, 2], and it is generally considered an important source of heating and acceleration of plasma particles. In particular, the kinetic Alfvén wave (KAW) turbulence is believed to play a major role in particle energization [3, 4, 5, 6, although all the other components of the low frequency spectrum have been studied in this respect, such as the Magnetosonic/whistler (MSW) mode [7, 8], the kinetic slow (KS) mode (or ion acoustic mode) [9, 10, and the ion-Bernstein wave (IBW) [11]. The so-called higher-order modes (HOM) have also been considered [12], but the detailed physics of the kinetic dissipation is not clearly understood.

In many laboratory and space plasmas, the effect of discrete charged particles (with finite kinetic energy) appears as spontaneously emitted electromagnetic (EM) fluctuations. The study of spontaneous emission is particularly useful in plasma diagnostics [13, 14, 15, 16]. First theoretical approaches have been developed for standard (bi-) Maxwellian representation of particle velocity distributions. The Alfvéncyclotron EM emissions from plasmas with stable distributions are found to have a significant contribution [5] to the observed magnetic field fluctuations in the solar wind at $1 \mathrm{AU}$ [17]. Moreover, Alfvén waves can be generated through a spontaneous process by the heavy ions which play critical roles in the solar transition region [18, 19]. Ref. [20] provides the steady-state spectra of low-frequency EM fluctuations of the Alfvénic type for thermal equilibrium plasma, and Ref. [21] explains the structure of low-frequency waves in thermal plasmas through hybrid simulations and spontaneous emission theory [22, 23]. Recently, a theory of spontaneously emitted EM fluctuations in thermal magnetized plasmas was formulated, for an arbitrary wave vector orientation and angular frequency, as well as for arbitrary polarization, on the basis of the Klimontovich plasma kinetic theory [22, 23].

Suprathermal populations are ubiquitous in various plasma systems including the fusion devices [24, 25], and especially the space plasma environments such as planetary magnetospheres and the solar wind. In the presence of suprathermals the high-energy tails of the velocity distributions are modelled by the Kappa power-laws [26, 27]. Kim et al. [28] generalize the results in Refs. 22, 23] in order to formulate the general spontaneous emission theory of EM fluctuations for the Kappa distributed plasmas, and a specific application was made to evaluate the high frequency emissions.

In the present paper, we compute the low-frequency EM fluctuations in Kappa distributed plasmas. The analysis is based upon recently formulated theory of spontaneously emitted EM fluctuations [28, which is overviewed in section 2. In Section 3 we test the low frequency approach by comparing with PIC simulations under an unrealistic ion-electron mass ratio condition that is subject to computational limitations. In Section 4 we apply the theory to low frequency regime with a realistic ionelectron mass ratio, and analyze the spontaneous emissions for each normal mode, and their variation with $\kappa_{i}$ and $\kappa_{e}$. These results are particularly important to understand 
the effects of suprathermal ions and electrons on the low frequency emissions. In Section 5 we summarize the findings and discuss on potential applications of the present investigation.

\section{OVERVIEW OF SPONTANEOUS EMISSION THEORY FOR KAPPA MAGNETIZED PLASMAS}

We consider magnetized plasmas described by the bi-Kappa velocity distribution function

$F_{a}\left(v_{\perp}, v_{\|}\right)=\frac{1}{\pi^{3 / 2}} \frac{1}{\Theta_{\perp a}^{2} \Theta_{\| a}} \frac{\Gamma\left(\kappa_{a}+1\right)}{\kappa_{a}^{3 / 2} \Gamma\left(\kappa_{a}-1 / 2\right)}\left[1+\frac{v_{\|}^{2}}{\kappa_{a} \Theta_{\| a}^{2}}+\frac{v_{\perp}^{2}}{\kappa_{a} \Theta_{\perp a}^{2}}\right]^{-\left(\kappa_{a}+1\right)}$,

which is valid for $\kappa_{a}>3 / 2$, and $\Gamma(z)$ is the gamma function. The quantities $\Theta_{\| a}$ and $\Theta_{\perp a}$ are proportional to the parallel and perpendicular thermal speeds, $v_{\| a}$ and $v_{\perp a}$, respectively,

$$
\Theta_{\| a}=\left(\frac{\kappa_{a}-3 / 2}{\kappa_{a}}\right)^{1 / 2} v_{\| a}, \quad \Theta_{\perp a}=\left(\frac{\kappa_{a}-3 / 2}{\kappa_{a}}\right)^{1 / 2} v_{\perp a} .
$$

where

$$
v_{\| a}=\left(\frac{2 T_{\| a}}{m_{a}}\right)^{1 / 2}, \quad v_{\perp a}=\left(\frac{2 T_{\perp a}}{m_{a}}\right)^{1 / 2} .
$$

The subscript $a$ denotes particle species, and $m_{a}$ and $T_{a}$ are mass and temperature for each species. According to Ref. [28], magnetic field fluctuation spontaneously emitted by Kappa magnetized plasma is given by

$$
\begin{aligned}
& \left\langle\delta B^{2}\right\rangle_{\mathbf{k}, \omega}=\frac{1}{|\Lambda|^{2}} \frac{c^{2} k^{2}}{\omega^{2}}\left\{\left|a_{12}\right|^{2} K_{11}+\left|a_{22}\right|^{2} K_{22}+\left|a_{23}\right|^{2} K_{33}\right. \\
& -\left(a_{12} a_{22}^{*}-a_{12}^{*} a_{22}\right) K_{12}-\left(a_{12} a_{23}^{*}+a_{12}^{*} a_{23}\right) K_{13}+\left(a_{22} a_{23}^{*}-a_{22}^{*} a_{23}\right) K_{23} \\
& +\frac{k_{\|}^{2}}{k^{2}}\left[\left|a_{11}\right|^{2} K_{11}+\left|a_{12}\right|^{2} K_{22}+\left|a_{13}\right|^{2} K_{33}\right. \\
& \left.+\left(a_{11} a_{12}^{*}-a_{11}^{*} a_{12}\right) K_{12}+\left(a_{11} a_{13}^{*}+a_{11}^{*} a_{13}\right) K_{13}+\left(a_{12} a_{13}^{*}-a_{12}^{*} a_{13}\right) K_{23}\right] \\
& +\frac{k_{\perp}^{2}}{k^{2}}\left[\left|a_{13}\right|^{2} K_{11}+\left|a_{23}\right|^{2} K_{22}+\left|a_{33}\right|^{2} K_{33}\right. \\
& \left.-\left(a_{13} a_{23}^{*}-a_{13}^{*} a_{23}\right) K_{12}+\left(a_{13} a_{33}^{*}+a_{13}^{*} a_{33}\right) K_{13}+\left(a_{23} a_{33}^{*}-a_{23}^{*} a_{33}\right) K_{23}\right] \\
& -\frac{k_{\|} k_{\perp}}{k^{2}}\left[\left(a_{11} a_{13}^{*}+a_{11}^{*} a_{13}\right) K_{11}-\left(a_{12} a_{23}^{*}+a_{12}^{*} a_{23}\right) K_{22}+\left(a_{13} a_{33}^{*}+a_{13}^{*} a_{33}\right) K_{33}\right. \\
& -\left(a_{11} a_{23}^{*}-a_{11}^{*} a_{23}+a_{12} a_{13}^{*}-a_{12}^{*} a_{13}\right) K_{12}+\left(a_{11} a_{33}^{*}+a_{11}^{*} a_{33}+2\left|a_{13}\right|^{2}\right) K_{13} \\
& \left.\left.+\left(a_{13} a_{23}^{*}-a_{13}^{*} a_{23}+a_{12} a_{33}^{*}-a_{12}^{*} a_{33}\right) K_{23}\right]\right\},
\end{aligned}
$$

where

$$
\Lambda=\Lambda_{11} \Lambda_{22} \Lambda_{33}+\Lambda_{12}^{2} \Lambda_{33}+\Lambda_{11} \Lambda_{23}^{2}-\Lambda_{22} \Lambda_{13}^{2}+2 \Lambda_{12} \Lambda_{23} \Lambda_{13},
$$

is the determinant of the linear dielectric response tensor, and

$$
a_{11}=\Lambda_{22}^{*} \Lambda_{33}^{*}+\left(\Lambda_{23}^{*}\right)^{2}
$$




$$
\begin{aligned}
& a_{12}=\Lambda_{13}^{*} \Lambda_{23}^{*}+\Lambda_{12}^{*} \Lambda_{33}^{*}, \\
& a_{13}=\Lambda_{12}^{*} \Lambda_{23}^{*}-\Lambda_{13}^{*} \Lambda_{22}^{*}, \\
& a_{22}=\Lambda_{11}^{*} \Lambda_{33}^{*}-\left(\Lambda_{13}^{*}\right)^{2}, \\
& a_{23}=\Lambda_{12}^{*} \Lambda_{13}^{*}+\Lambda_{11}^{*} \Lambda_{23}^{*}, \\
& a_{33}=\Lambda_{11}^{*} \Lambda_{22}^{*}+\left(\Lambda_{12}^{*}\right)^{2},
\end{aligned}
$$

are elements of cofactor matrix associated with the inverse dielectric response tensor. The linear dielectric response tensor for bi-Kappa VDFs is given by [28, 29, 30]

$$
\begin{aligned}
& \Lambda_{11}=1-\frac{c^{2} k_{\|}^{2}}{\omega^{2}}+\sum_{a} \frac{\omega_{p a}^{2}}{\omega^{2}} \sum_{n=-\infty}^{\infty} \frac{n^{2}}{\mu_{a}}\left[\xi_{a}^{0} Z_{n, \kappa_{a}}^{(1,2)}\left(\mu_{a}, \xi_{a}^{n}\right)+\frac{1}{2} A_{a} \frac{\partial}{\partial \xi_{a}^{n}} Z_{n, \kappa_{a}}^{(1,1)}\left(\mu_{s}, \xi_{a}^{n}\right)\right], \\
& \Lambda_{22}=1-\frac{c^{2} k^{2}}{\omega^{2}}+\sum_{a} \frac{\omega_{p a}^{2}}{\omega^{2}} \sum_{n=-\infty}^{\infty}\left[\xi_{a}^{0} W_{n, \kappa_{a}}^{(1,2)}\left(\mu_{a}, \xi_{a}^{n}\right)+\frac{1}{2} A_{a} \frac{\partial}{\partial \xi_{a}^{n}} W_{n, \kappa_{a}}^{(1,1)}\left(\mu_{a}, \xi_{a}^{n}\right)\right], \\
& \Lambda_{12}=i \sum_{a} \frac{\omega_{p a}^{2}}{\omega^{2}} \sum_{n=-\infty}^{\infty} n\left[\xi_{a}^{0} \frac{\partial}{\partial \mu_{a}} Z_{n, \kappa_{a}}^{(1,2)}\left(\mu_{a}, \xi_{a}^{n}\right)+\frac{1}{2} A_{a} \frac{\partial^{2}}{\partial \mu_{a} \partial \xi_{a}^{n}} Z_{n, \kappa_{a}}^{(1,1)}\left(\mu_{a}, \xi_{a}^{n}\right)\right], \\
& \Lambda_{13}=\frac{c^{2} k_{\perp} k_{\|}}{\omega^{2}}-\sum_{a} \frac{\omega_{p a}^{2}}{\omega^{2}} \frac{\Theta_{\| a}}{\Theta_{\perp a}} \sum_{n=-\infty}^{\infty} \frac{n \Omega_{a}}{k_{\perp} \Theta_{\perp a}}\left(\xi_{a}^{0}-A_{a} \xi_{a}^{n}\right) \frac{\partial}{\partial \xi_{a}^{n}} Z_{n, \kappa_{a}}^{(1,1)}\left(\mu_{a}, \xi_{a}^{n}\right), \\
& \Lambda_{33}=i \sum_{a} \frac{\omega_{p a}^{2}}{\omega^{2}} \frac{\Theta_{\| a}}{\Theta_{\perp a}} \frac{k_{\perp} \Theta_{\perp a}}{2 \Omega_{a}} \sum_{n=-\infty}^{\infty}\left(\xi_{a}^{0}-A_{a} \xi_{a}^{n}\right) \frac{\partial^{2}}{\partial \mu_{a} \partial \xi_{a}^{n}} Z_{n, \kappa_{a}}^{(1,1)}\left(\mu_{a}, \xi_{a}^{n}\right), \\
& \omega^{2} k_{\perp}^{2}
\end{aligned}
$$

In Eq. (6), various quantities are defined by

$$
\begin{aligned}
& Z_{n, \kappa}^{(\alpha, \beta)}(\mu, \xi)=2 \int_{0}^{\infty} d x \frac{x J_{n}^{2}(\nu x)}{\left(1+x^{2} / \kappa\right)^{\lambda-1}} Z_{\kappa}^{(\alpha, \beta)}\left(\frac{\xi}{\sqrt{1+x^{2} / \kappa}}\right) \\
& Y_{n, \kappa}^{(\alpha, \beta)}(\mu, \xi)=\frac{2}{\mu} \int_{0}^{\infty} d x \frac{x^{3} J_{n-1}(\nu x) J_{n+1}(\nu x)}{\left(1+x^{2} / \kappa\right)^{\lambda-1}} Z_{\kappa}^{(\alpha, \beta)}\left(\frac{\xi}{\sqrt{1+x^{2} / \kappa}}\right) \\
& W_{n, \kappa}^{(\alpha, \beta)}(\mu, \xi)=\frac{n^{2}}{\mu} Z_{n, \kappa}^{(\alpha, \beta)}(\mu, \xi)-2 \mu Y_{n, \kappa}^{(\alpha, \beta)}(\mu, \xi), \\
& Z_{\kappa}^{(\alpha, \beta)}(\xi)=\frac{1}{\sqrt{\pi} \kappa^{\beta+1 / 2}} \frac{\Gamma(\lambda-1)}{\Gamma(\sigma-3 / 2)} \int_{-\infty}^{\infty} d s \frac{\left(1+s^{2} / \kappa\right)^{-(\lambda-1)}}{s-\xi}, \quad \text { for } \operatorname{Im}(\xi)>0 \\
& \mu_{a}=k_{\perp}^{2} \rho_{a}^{2}, \quad \rho_{a}^{2}=\frac{\Theta_{\perp a}^{2}}{2 \Omega_{a}^{2}}, \quad \nu_{a}^{2}=2 \mu_{a}, \quad \xi_{a}^{n}=\frac{\omega-n \Omega_{a}}{k_{\|} \Theta_{\| a}}, \\
& A_{a}=1-\frac{\Theta_{\perp a}^{2}}{\Theta_{\| a}^{2}}, \quad \lambda=\sigma+\beta, \quad \sigma=\kappa+\alpha .
\end{aligned}
$$

Here, $\alpha, \beta, \sigma$, and $\lambda$ are real numbers. $Z_{n, \kappa}^{(\alpha, \beta)}(\mu, \xi)$ and $Y_{n, \kappa}^{(\alpha, \beta)}(\mu, \xi)$ are two-variables kappa plasma functions (2VKPs), and $Z_{\kappa}^{(\alpha, \beta)}(\xi)$ is the Kappa plasma dispersion function $(\kappa \mathrm{PDF})$, which is defined for positive imaginary part of the argument. For arguments with negative imaginary part, the $\kappa \mathrm{PDF}$ must be continued analytically. $J_{n}$ is the 
Bessel function of the first kind of order $n$, the quantity $\rho_{a}$ is the gyroradius, $\mu_{a}$ is the normalized gyroradius of particle $a$, and $A_{a}$ is the thermal anisotropy parameter. The detailed derivation of formulas and properties of above special functions can be found in Refs. [29, 30]. The source electric field fluctuations in the bi-Kappa plasma, immersed in a constant magnetic field, are given by [28]

$$
\begin{aligned}
& K_{11}=\pi^{1 / 2} \sum_{a} \frac{\sqrt{T_{\| a} T_{\perp a}}}{2 \pi^{3} \omega} \frac{\omega_{p a}^{2}}{\omega^{2}}\left(\frac{\kappa_{a}-3 / 2}{\kappa_{a}}\right) g\left(\kappa_{a}\right) \\
& \times \sum_{n=-\infty}^{\infty} \frac{\Theta_{\perp a}}{\Theta_{\| a}} \frac{n^{2}}{\mu_{a}}\left[1+\frac{\left(\xi_{a}^{n}\right)^{2}}{\kappa_{a}}\right]^{-\kappa_{a}} \xi_{a}^{0} H_{n, \kappa_{a}}^{(1,1)}\left[\frac{\left(z_{a}^{n}\right)^{2}}{2}\right], \\
& K_{12}=i \pi^{1 / 2} \sum_{a} \frac{\sqrt{T_{\| a} T_{\perp a}}}{2 \pi^{3} \omega} \frac{\omega_{p a}^{2}}{\omega^{2}}\left(\frac{\kappa_{a}-3 / 2}{\kappa_{a}}\right) g\left(\kappa_{a}\right) \\
& \times \sum_{n=-\infty}^{\infty} n \frac{\Theta_{\perp a}}{\Theta_{\| a}}\left[1+\frac{\left(\xi_{a}^{n}\right)^{2}}{\kappa_{a}}\right]^{-\kappa_{a}} \xi_{a}^{0} \frac{\partial}{\partial \mu_{a}} H_{n, \kappa_{a}}^{(1,1)}\left[\frac{\left(z_{a}^{n}\right)^{2}}{2}\right], \\
& K_{13}=\pi^{1 / 2} \sum_{a} \frac{\sqrt{T_{\| a} T_{\perp a}}}{2 \pi^{3} \omega} \frac{\omega_{p a}^{2}}{\omega^{2}}\left(\frac{\kappa_{a}-3 / 2}{\kappa_{a}}\right) g\left(\kappa_{a}\right) \\
& \times \sum_{n=-\infty}^{\infty} \frac{k_{\perp} \Theta_{\perp a}}{\Omega_{a}} \frac{n}{\mu_{a}} \xi_{a}^{n}\left[1+\frac{\left(\xi_{a}^{n}\right)^{2}}{\kappa_{a}}\right]^{-\kappa_{a}} \xi_{a}^{0} H_{n, \kappa_{a}}^{(1,1)}\left[\frac{\left(z_{a}^{n}\right)^{2}}{2}\right], \\
& K_{22}=\pi^{1 / 2} \sum_{a} \frac{\sqrt{T_{\| a} T_{\perp a}}}{2 \pi^{3} \omega} \frac{\omega_{p a}^{2}}{\omega^{2}}\left(\frac{\kappa_{a}-3 / 2}{\kappa_{a}}\right) g\left(\kappa_{a}\right) \\
& \times \sum_{n=-\infty}^{\infty} 2 \frac{\Theta_{\perp a}}{\Theta_{\| a}}\left[1+\frac{\left(\xi_{a}^{n}\right)^{2}}{\kappa_{a}}\right]^{-\left(\kappa_{a}-1\right)} \xi_{a}^{0} \tilde{H}_{n, \kappa_{a}}^{(1,1)}\left[\frac{\left(z_{a}^{n}\right)^{2}}{2}\right] \\
& K_{23}=-i \pi^{1 / 2} \sum_{a} \frac{\sqrt{T_{\| a} T_{\perp a}}}{2 \pi^{3} \omega} \frac{\omega_{p a}^{2}}{\omega^{2}}\left(\frac{\kappa_{a}-3 / 2}{\kappa_{a}}\right) g\left(\kappa_{a}\right) \\
& \times \sum_{n=-\infty}^{\infty} \frac{k_{\perp} \Theta_{\perp a}}{\Omega_{a}} \xi_{a}^{n}\left[1+\frac{\left(\xi_{a}^{n}\right)^{2}}{\kappa_{a}}\right]^{-\kappa_{a}} \xi_{a}^{0} \frac{\partial}{\partial \mu_{a}} H_{n, \kappa_{a}}^{(1,1)}\left[\frac{\left(z_{a}^{n}\right)^{2}}{2}\right], \\
& K_{33}=\pi^{1 / 2} \sum_{a} \frac{\sqrt{T_{\| a} T_{\perp a}}}{2 \pi^{3} \omega} \frac{\omega_{p a}^{2}}{\omega^{2}}\left(\frac{\kappa_{a}-3 / 2}{\kappa_{a}}\right) g\left(\kappa_{a}\right) \\
& \times \sum_{n=-\infty}^{\infty} 2 \frac{\Theta_{\| a}}{\Theta_{\perp a}}\left(\xi_{a}^{n}\right)^{2}\left[1+\frac{\left(\xi_{a}^{n}\right)^{2}}{\kappa_{a}}\right]^{-\kappa_{a}} \xi_{a}^{0} H_{n, \kappa_{a}}^{(1,1)}\left[\frac{\left(z_{a}^{n}\right)^{2}}{2}\right],
\end{aligned}
$$

where

$$
\begin{array}{ll}
H_{n, \kappa}^{(\alpha, \beta)}(z)=2 \int_{0}^{\infty} d x \frac{x J_{n}^{2}(y x)}{\left(1+x^{2} / \kappa\right)^{\lambda-1}}, & \tilde{H}_{n, \kappa}^{(\alpha, \beta)}(z)=2 \int_{0}^{\infty} d x \frac{x^{3} J_{n}^{\prime 2}(y x)}{\left(1+x^{2} / \kappa\right)^{\lambda-1}}, \\
2 z=y^{2}, & z_{a}^{n}=\nu_{a} \sqrt{1+\frac{\left(\xi_{a}^{n}\right)^{2}}{\kappa_{a}}}, \quad g\left(\kappa_{a}\right)=\frac{\Gamma\left(\kappa_{a}+1\right)}{\kappa_{a}^{3 / 2} \Gamma\left(\kappa_{a}-1 / 2\right)} .
\end{array}
$$

Here, the function $H_{n, \kappa}^{(\alpha, \beta)}(z)$ denotes the Kappa plasma gyroradius function ( $\left.\kappa \mathrm{PGF}\right)$, related to the particles' finite gyroradii when their probability distribution function is 
described by a Kappa VDF.

In the present paper, we consider an isotropic plasma temperature and focus on the case of fluctuations in the low-frequency range which include the kinetic Alfvén (KAW), fast magnetosonic/whistler (MSW), ion-acoustic (or kinetic slow mode) (KS), ion Bernstein cyclotron modes (IBW), and higher-order modes (HOM).

\section{TEST CASES FOR A COMPARISON WITH PIC SIMULATIONS OF SPONTANEOUS EMISSIONS IN THE LOW-FREQUENCY REGIME}

In order to test the validity of the approach in the low frequency regime in the present section we compare the EM spontaneous emissions predicted by the theory with those obtained from PIC simulations. Since a realistic mass ratio $m_{i} / m_{e}=1836$ would increase considerably the computational needs, here we perform a $1.5 \mathrm{D}$ (one spatial dimension and three dimensional velocity) full PIC simulations with a reduced mass ratio $m_{i} / m_{e}=25$. In order to resolve the proton scales, we also reduce the ratio between electron plasma frequency and electron cyclotron frequency to $\omega_{p e} /\left|\Omega_{e}\right|=2$, and use a simulation box of $L=1024 c / \omega_{p e}$ with 4096 grid points and 1000 particles per grid per species. The time step is $\omega_{p e} t=0.01$ and the simulation runs until $\omega_{p e} t_{\max }=20971.52$, wich correspond to $\Omega_{p} t_{\max }=419.43$.

Two cases are considered, firstly assuming Kappa distributed ions and Maxwellian electrons, and then, in the second case, Maxwellian ions and Kappa distributed electrons. In numerical examples to be discussed below, plasma beta is chosen to be $\beta_{i}=\beta_{e}=0.1$. In Figure 1 and 2 we plot spectra of the magnetic field fluctuation by using a color map scheme.

\subsection{Kappa distributed ions}

Firstly, we assume the Kappa distributed ions, since the low frequency fluctuations are mainly triggered by the ions. In Figure 1, we plot the normalized magnetic field fluctuation spectra obtained from the theory (top panels) and PIC simulations (bottom panels), for three different magnetic field inclination angles $\theta=10^{\circ}, 70^{\circ}$, and $85^{\circ}$. We also choose two different values for the ion power index $\kappa_{i}=2$, and 5 . In general, it is known that the spontaneously emitted EM fluctuations are enhanced in the near vicinity of the plasma normal modes, that is, for angular frequency $\omega$ satisfying the dispersion relations, $\omega=\omega(\mathbf{k})$. Figure 1 indeed shows such enhancements in the near vicinity of the plasma normal modes. The representative low frequency eigenmodes are indicated in Figure 1. The left column panels correspond to $\theta=10^{\circ}$ (quasi-parallel case), and spontaneously emitted fluctuations are highly enhanced in the vicinities of two quasi-parallel normal modes, i.e., Alfvén/ion-cyclotron (AIC) and MSW branches. For larger angle, e.g., $\theta=70^{\circ}$, (middle column panels), the emission pattern begins the transition from the AIC/MSW dual mode structure to a more complicated form possessing multiple-harmonic ion-Bernstein bands. For $\theta=85^{\circ}$, the right column 

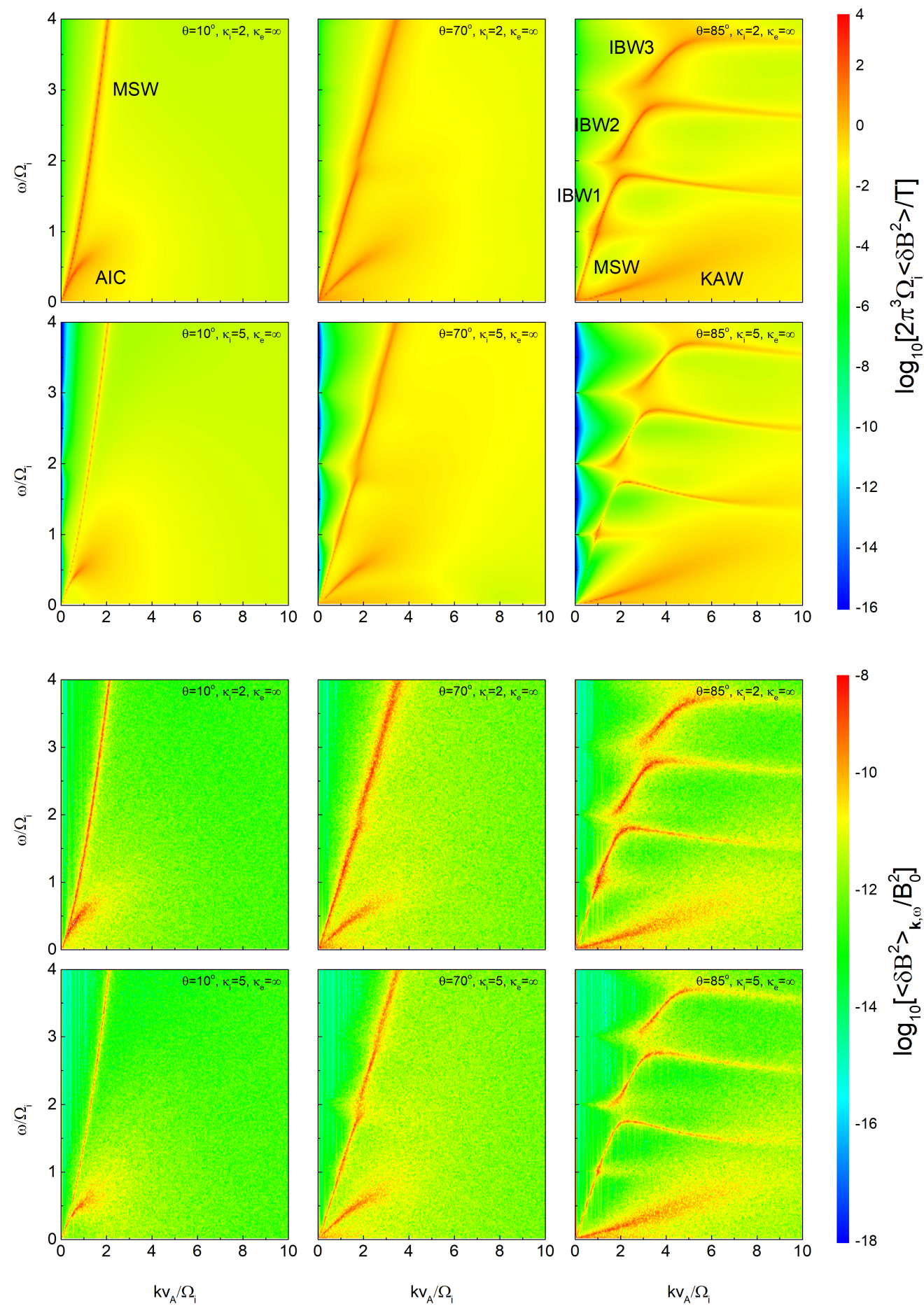

Figure 1. Normalized magnetic fluctuation spectra from the theory (top panels) and simulations (bottom panels) for different angles $\theta=10^{\circ}, 70^{\circ}$, and $85^{\circ}$, and different $\kappa_{i}=2,5\left(m_{i} / m_{e}=25, \omega_{p e} /|\Omega e|=2, \kappa_{e} \rightarrow \infty\right.$ and $\left.\beta_{e}=\beta_{i}=0.1\right)$. 
panels of Figure 1, (quasi-perpendicular case), show that the ion Bernstein mode at each harmonic appears as a breakup of the fast MSW mode at larger propagation angles. It shows the transition from the AIC mode to KAW and KS (ion-acoustic) mode. The KS mode for $\theta=10^{\circ}$ does not show up in the magnetic fluctuation spectrum, because the mode is quasi-electrostatic for small $\theta$. For large $\theta$, however, The KS mode appear in the magnetic fluctuations, because a compressional magnetic field perturbation is obtained in the KS mode, but for the present low beta case the mode has a low frequency, so it does not appear to be obvious. The case of $\theta=85^{\circ}$ also shows enhancements at $\omega=n \Omega_{i}$ which are associated with non-collective modes. When $\kappa_{i}=2$, non-collective fluctuations are not clear because of the background noise, but these are more clear when $\kappa_{i}=5$. Non-collective fluctuations turn out to be constrained by the least damped HOM, because HOMs are heavily damped modes and they define the region in which non-collective fluctuations exist [5, 12, 31].

Note the excellent agreement between the theory (top panels) and the simulations (bottom panels). The power in this spectra is obviously concentrated in the normal modes and the case with $\kappa_{i}=2$ shows higher intensities than the case with $\kappa_{i}=5$ in both the results from the simulation and theory. Notice that normalizations are different, that is why the intensities are not the same. The simulated magnetic field is normalized to the background magnetic field, so the spectra obtained from PIC simulations correspond to the power $\delta E^{2} / B_{0}^{2}$ in bottom panels. On the other hand, the spectra computed from theory are normalized as $2 \pi^{3} \Omega_{i} \delta B^{2} / T$.

\subsection{Kappa distributed electrons}

Figure 2 displays the spontaneous emissions for a plasma with Kappa distributed electrons and Maxwellian ions. We compare the EM fluctuations obtained from the theory and PIC simulations, top and bottom panels, respectively. All parameters and formats are the same as in Figure 1, except that $\kappa_{i} \rightarrow \infty$ and we assume two distinct cases corresponding to $\kappa_{e}=2$, and 5 . Spontaneous emissions show enhancements at each normal mode, and spectral intensities vary with $\kappa_{e}$. Thus, for $\kappa_{e}=2$ the intensities are visibly higher than those obtained for $\kappa_{e}=5$, and the results in Figure 2 show, in general, a good agreement between theory and simulations.

Overall, from these comparative analyses we can conclude that PIC simulations confirm the validity of our theoretical approach. Also obvious are the effects of suprathermal electrons as well as suprathermal ions on the spontaneous fluctuations in the low frequency regime, and these effects are explored further in the next section.

\section{EFFECTS OF SUPRATHERMAL PARTICLES ON LOW-FREQUENCY EMISSIONS}

In the previous section we have tested the validity of our theoretical approach for the low frequency EM fluctuations by a comparison with PIC simulations, and we found 

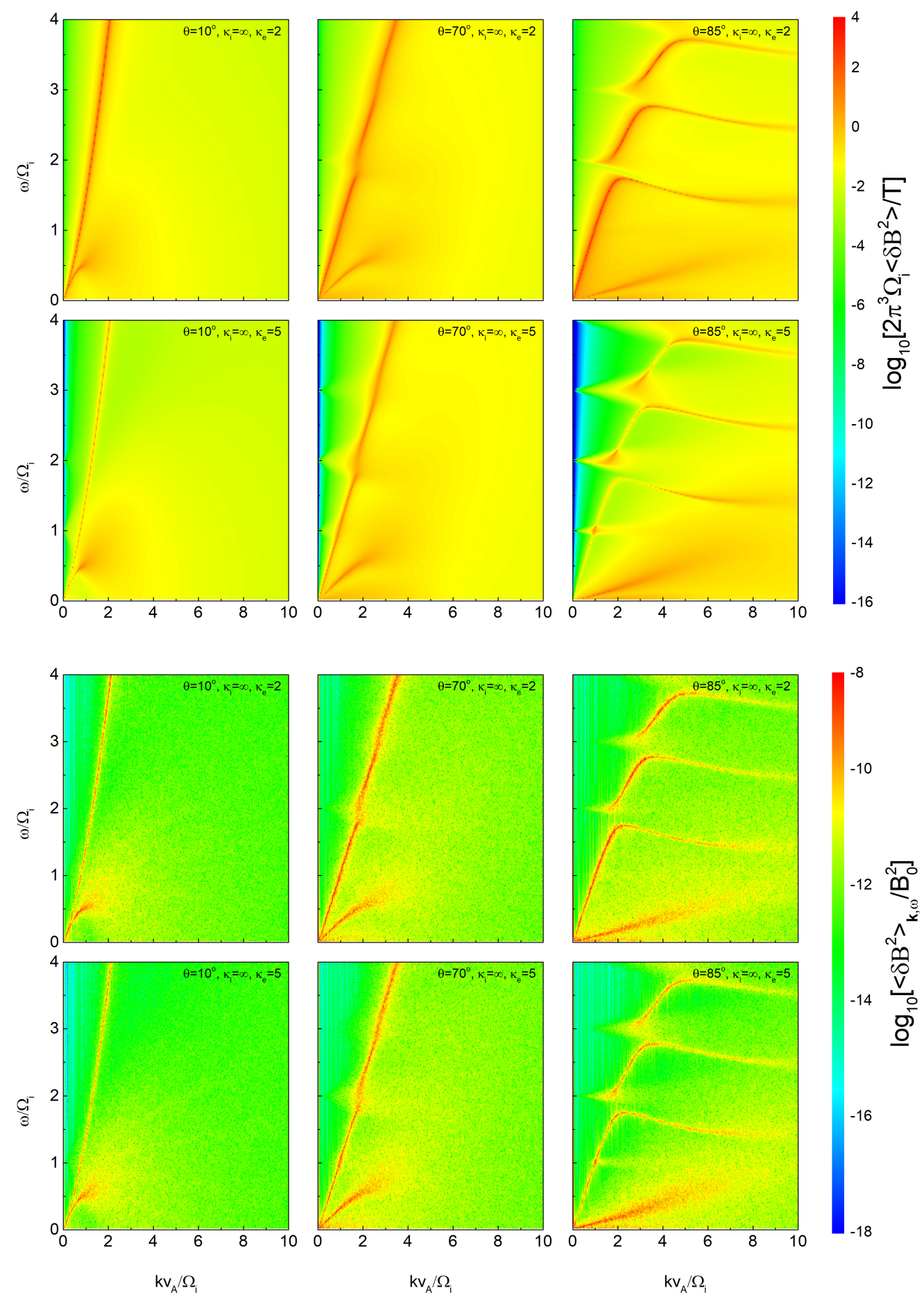

Figure 2. The same as in Figure 1 but for $\kappa_{i} \rightarrow \infty$ and $\kappa_{e}=2,5$.

a good agreement between theory and simulations. The current section presents the results from computations with a realistic mass ratio $m_{i} / m_{e}=1836$, and describes the effects of suprathermal ions and electrons on the low frequency fluctuations by varying $\kappa_{i}$ and $\kappa_{e}$, respectively. Thus, for the $\kappa$-index (either for $\kappa_{i}$ or $\kappa_{e}$ ) we consider three different values $\kappa=2,5$ and 10 , and three different magnetic field inclination angles 
$\theta=5^{\circ}, 70^{\circ}$, and $88^{\circ}$. In order to compute magnetic field fluctuations we set the Alfvén velocity to $v_{A} / c=10^{-3}$, and plasma beta is chosen to be $\beta_{i}=\beta_{e}=8 \pi n T / B^{2}=0.1$.

\subsection{Effects of Suprathermal ions}

To delineate the effects of suprathermal ions, we consider them Kappa distributed and Maxwellian electrons $\left(\kappa_{e}=\infty\right)$. Figure 3 shows variation of the normalized magnetic field fluctuation spectra with $\kappa_{i}$ and $\theta$. As with the results from the previous section, the fluctuation spectra show enhancements in the near vicinity of normal plasma modes. In general, the EM fluctuations strengthen as $\kappa_{i}$ decreases (with the ion temperature assumed constant, independent of $\kappa_{i}$ ), suggesting that these emissions are stimulated by the suprathermal ions in the tail of the velocity distribution function (VDF). An explanation can be provided by the resonant particles, assuming at work the Landau or cyclotron (linear) interactions between particles and the associated normal modes. We estimate the resonant conditions (velocities) at different points, e.g., A, B, C, D and E, corresponding to different plasma modes in Figure 3 (top panels). In quasi-parallel case $\left(\theta=5^{\circ}\right)$, points $\mathrm{A}$ and $\mathrm{B}$ correspond to AIC and MSW branches, respectively, while in quasi-perpendicular case $\left(\theta=88^{\circ}\right)$, points $\mathrm{C}$ and $\mathrm{D}$ are related with KAW and MSW, respectively, and point $\mathrm{E}$ can be associated with IBW. Resonant particles satisfy

$$
\omega-\mathbf{k} \cdot \mathbf{v}=n \Omega, \quad n=0, \pm 1, \pm 2, \cdots,
$$

where the harmonic number $n=0$ for Landau resonance, and $n \neq 0$ for cyclotron resonance.

For the quasi-parallel case $\left(k_{\perp} \rightarrow 0\right)$, the resonant speed becomes $v_{\|, r e s}^{(n)}=(\omega-n \Omega) / k$ with $n \neq 0$. The emissions related to AIC (point A) and MSW (point B) strengthen as $\kappa_{i}$-index decreases. The ion-cyclotron (AIC) mode is left-handed (LH) circularly polarized and the ions undergo normal cyclotron resonance, when the particle velocity $(\mathbf{v})$ and the wave propagation (given by the wave-vector, $\mathbf{k}$ ) are opposite, i.e, $\mathrm{n}=1$. On the other hand, the whistler (MSW) mode is right-handed ( $\mathrm{RH}$ ) circularly polarized, and the ions undergo anomalous cyclotron resonance, when particles and waves travel in the same direction, i.e, $\mathrm{n}=-1$. Figure 4 (a) represents (normalized) ion-Kappa parallel VDFs (solid lines) for $\kappa_{i}=2,5$ and 10, and (normalized) resonant velocities of ions at the points A and B (dashed vertical lines), which are defined as

$$
F_{i}\left(V_{\|}\right) \equiv 2 \pi \int_{0}^{\infty} v_{\perp} d v_{\perp} F_{i}\left(v_{\|}, v_{\perp}\right), \quad V_{\|, r e s, i}^{(n)} \equiv \frac{v_{\|, r e s, i}^{(n)}}{c}
$$

For both points A and B the resonant speeds are located in the tail of ion Kappa-VDFs, meaning that the number of resonant particles increase as $\kappa_{i}$ decreases, and this may explain why emissions related with AIC and MSW strengthen as $\kappa_{i}$ decreases at these points.

Similarly, for the quasi-perpendicular case, dominant is the Landau $(n=0)$ resonance, and the resonant speed becomes $v_{\perp, \text { res }}^{(0)}=\omega / k$ [3]. Normalized ion-Kappa perpendicular VDFs (solid lines) for $\kappa_{i}=2,5$ and 10, and the Landau resonant velocities 

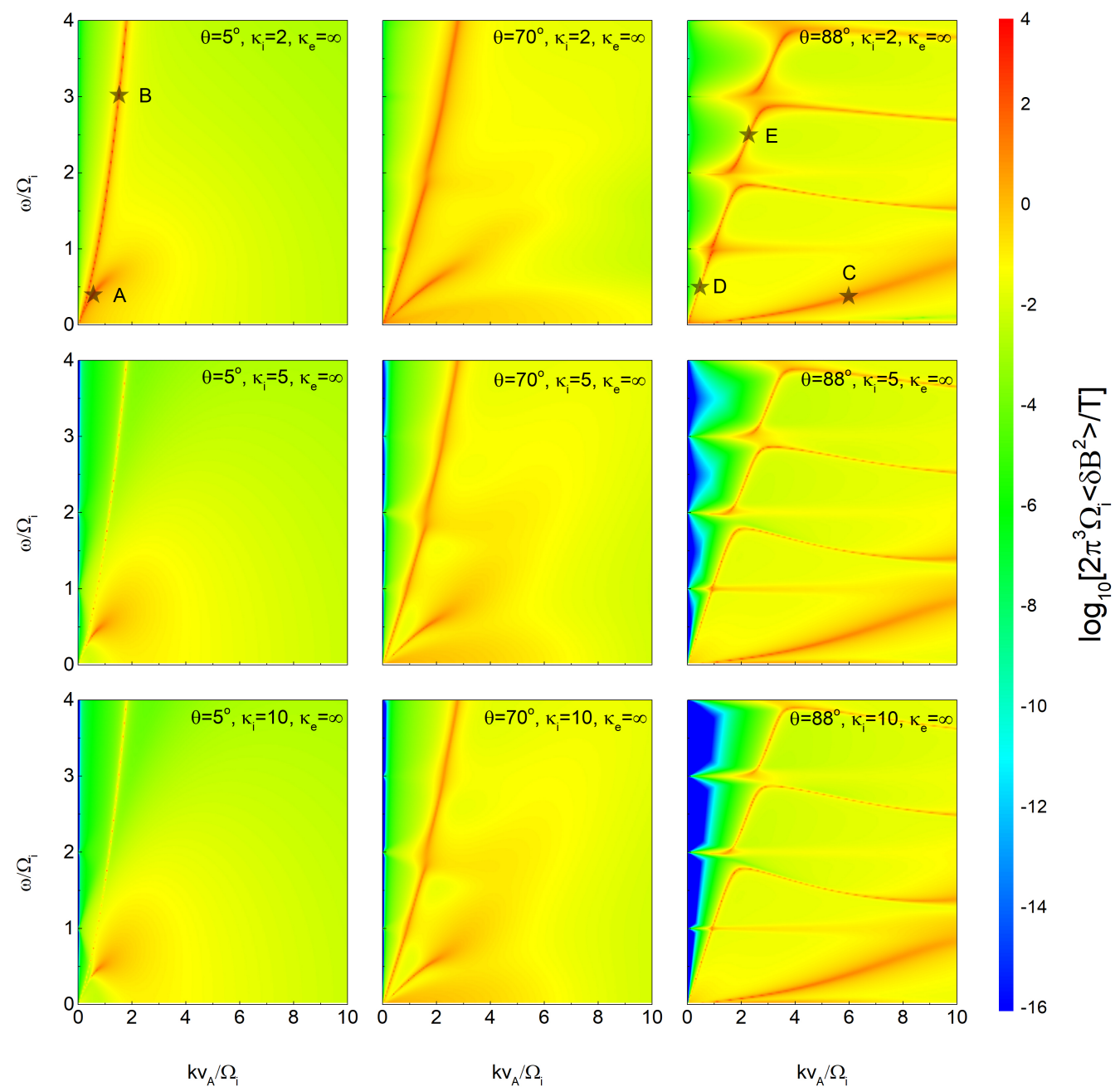

Figure 3. Normalized fluctuation spectra obtained for different angles $\theta=5^{\circ}, 70^{\circ}$, and $88^{\circ}$, and different $\kappa_{i}=2$ (top), 5 (middle) and 10 (bottom). Plasma parameters: $m_{i} / m_{e}=1836, v_{A} / c=10^{-3}, \kappa_{e} \rightarrow \infty$ and $\beta_{i}=\beta_{e}=0.1$.

at points $\mathrm{C}, \mathrm{D}$ and $\mathrm{E}$ (dashed vertical lines) are displayed in Figure $4(\mathrm{~b}) . F_{i}\left(V_{\perp}\right)$ and $V_{\perp, r e s, i}^{(0)}$ are defined as

$$
F_{i}\left(V_{\perp}\right) \equiv \int_{-\infty}^{\infty} d v_{\|} F_{i}\left(v_{\|}, v_{\perp}\right), \quad V_{\perp, r e s, i}^{(0)} \equiv \frac{v_{\perp, r e s, i}^{(0)}}{c} .
$$

Only very slow particles, with very low energies, can be resonant with KAW (point C), while for MSW (point D) and IBW (point E) the resonant speeds are found in the tail of ion Kappa-VDFs. In Figure 3, point $\mathrm{C}$ does not show a noticeable change in intensity when $\kappa_{i}$ decreases. In this case the number of particles that can interact with KAWs is larger when $\kappa_{i}=2$, see Figure 4 (b), but these populations have very low energies, and their (stimulating) effect on the emissions is only minor. At points D and $\mathrm{E}$, spectral intensity increases when $\kappa_{i}$-index decreases, since more resonant particles 
are responsible for these emissions, see Figure 4 (b).
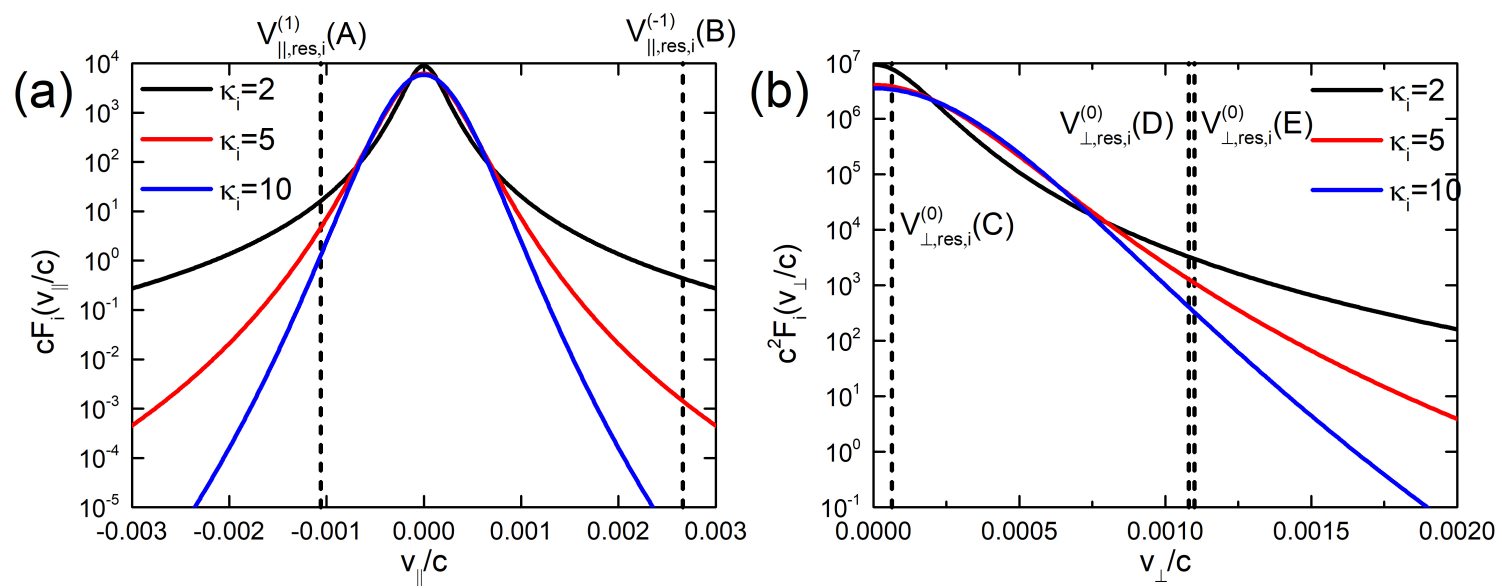

Figure 4. Normalized ion Kappa parallel (a) and perpendicular (b) velocity distributions and the resonant velocities at points A, B, C, D and E in Figure 3.

\subsection{Effects of Suprathermal Electrons}

The low-frequency waves and fluctuations are mainly triggered by the heavier species and the electrons are not expected to have a major influence, but here we show that theoretical results need to be adjusted in order to make them physically relevant. Much lighter than protons (ions), the electrons are not necessarily resonant with the low frequency fluctuations, but their presence may have an indirect effect changing, for instance, the dispersion properties and implicitly the number of resonant protons which directly trigger the fluctuations. Recent studies of parallel EM waves and instabilities have described these indirect effects of electrons [32, 33], and in particular those of suprathermal electrons [34, 35]. Perpendicular waves may be also affected by the electrons, which form a charge neutralising background and may therefore play a crucial shielding role that cannot be neglected [36, 37].

In order to study the effects of suprathermal electrons on the low-frequency, spontaneous emissions, here we consider Kappa distributed electrons and Maxwellian ions $\left(\kappa_{i}=\infty\right)$. Figure 5 displays the EM spontaneous emissions computed with a real mass ratio by varying $\kappa_{e}=2,5,10$, and $\theta=5^{\circ}, 70^{\circ}$, and $88^{\circ}$. Enhancements of the fluctuations are obtained in the near vicinity of plasma normal modes, and, in general, the intensity spectra strengthen as $\kappa_{e}$ decreases (with $T_{e}$ assumed independent of $\kappa_{e}$ ). In particular, most noticeable and intriguing at the same time, are the enhancements of AIC and MSW emissions in quasi-parallel direction $\left(\theta=5^{\circ}\right)$, i.e., points $\mathrm{A}$ and $\mathrm{B}$, respectively, despite the fact that the electrons do not interact resonantly with these fluctuations.

Figure 6 shows the electron Kappa VDFs, parallel component in panel (a) and perpendicular component in panel (b), and with dashed lines the resonant electron speeds at the same points A, B, C, D and E considered above are overplotted. For 

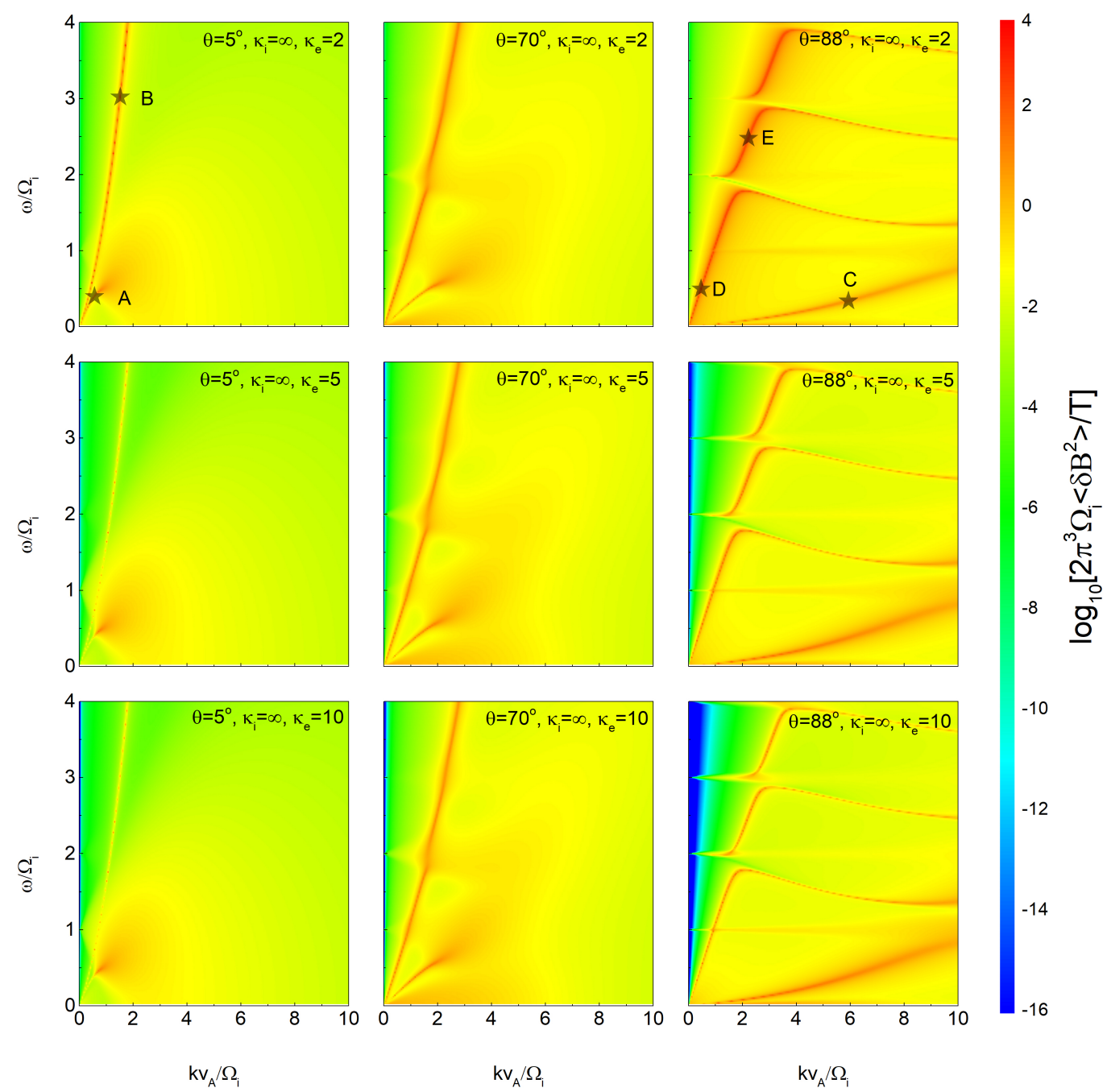

Figure 5. Normalized fluctuation spectra obtained for different angles $\theta=5^{\circ}, 70^{\circ}$, and $88^{\circ}$, and different $\kappa_{e}=2$ (top), 5 (middle) and 10 (bottom). Plasma parameters: $m_{i} / m_{e}=1836, v_{A} / c=10^{-3}, \kappa_{i} \rightarrow \infty$ and $\beta_{i}=\beta_{e}=0.1$.

the quasi-parallel fluctuations $\left(\theta=5^{\circ}\right.$ ), i.e., AIC (point A) and MSWs (point B), the resonant electron speed reduces to $v_{\|, \text {res }}^{(1)}=\left|\Omega_{e}\right| / k$ (since $\omega \ll\left|\Omega_{e}\right|$ ), and the resulting values (dashed lines) are simply unphysical, being greater than the speed of light in vacuum $v_{\|, \text {res }}^{(1)}>c$ (point B). Thus, the particle populations increase with decreasing $\kappa_{e}$, but the enhancing effect of the spectra intensity obtained in Figure 5 is purely mathematical, without a real physical meaning. The spontaneous emission formulae in the section 2 do not take into account the relativistic effects. A straightforward way to introduce these limitations and provide realistic evaluations of the spontaneous emissions would be to remove the cyclotron effects $(n \neq 0)$ of electrons from Eq. (8), and keep only the Landau resonance $(n=0)$ terms (which are terms of the source field fluctuations by the discrete particle effects). The EM fluctuations obtained with these 
corrections are shown in Figure 7 for $\kappa_{e}=2$. Comparing Figures 5 and 7 we can easily observe that the quasi-parallel modes are not affected by the suprathermal electrons, while their effects maintain for highly oblique wave-vectors. These results suggest that electrons may have an influence on the emissions except for quasi-parallel case.
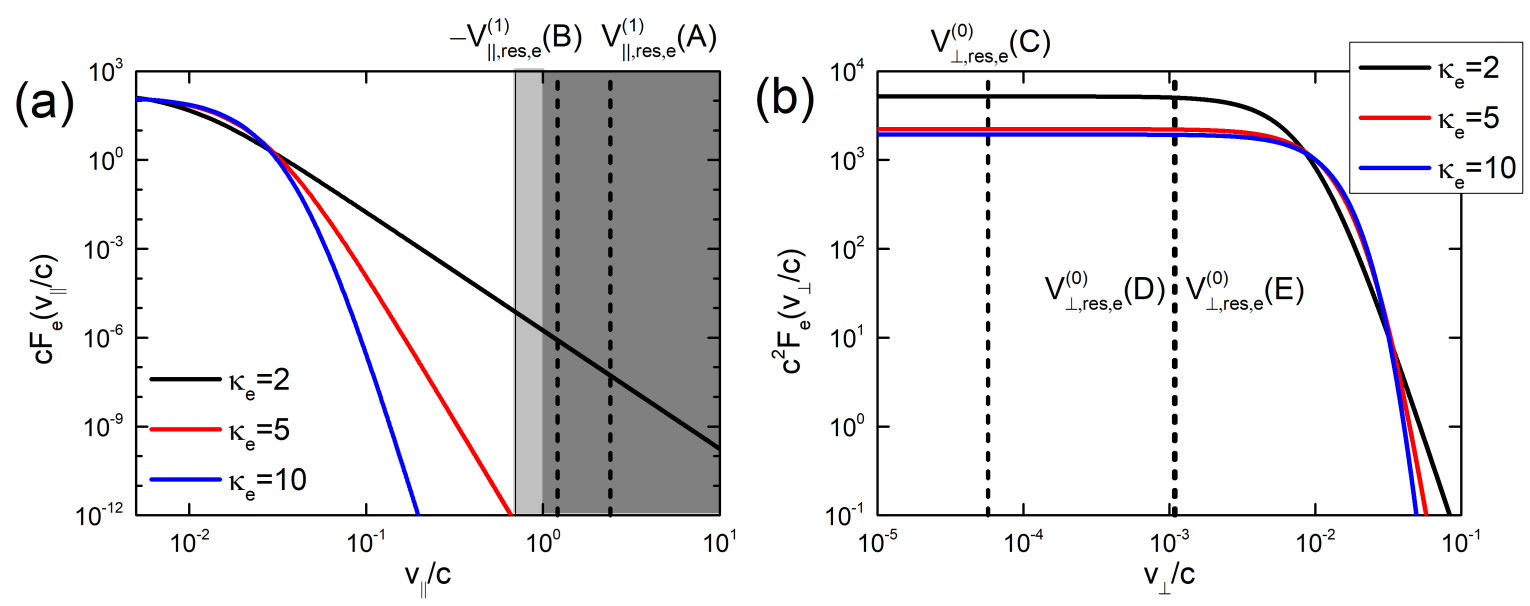

Figure 6. Normalized electron Kappa parallel (a) and perpendicular (b) velocity distributions for various $\kappa_{e}$, and the resonant velocities at points $\mathrm{A}, \mathrm{B}, \mathrm{C}, \mathrm{D}$ and $\mathrm{E}$ in Figure 3. Both of (a) and (b) are log-log scale plots.

For quasi-perpendicular wave-vectors $\left(\theta=88^{\circ}\right)$, we may consider Landau resonance only. All resonant speeds, corresponding to points C, D and E, are very low, near the peak of the electron distribution. The population of resonant particles increases with decreasing $\kappa_{e}$ suggesting a possible explanation for the enhancements of spontaneous emissions obtained at points D and E in Figure 5. However, for KAWs this explanation does not apply, since at point $\mathrm{C}$ the intensity of fluctuations show an opposite influence, slightly decreasing with decreasing $\kappa_{e}$. Indeed, we should not expect a major implication of these electron populations, which have low and very low energies, but other indirect effects, as the ones discussed above, may be at work in this case. The existing studies of low-frequency waves in perpendicular directions restrict only to the effects of suprathermal protons (ions). Our present results suggest additional effects of suprathermal electrons, which need to be decoded by the future investigations.

\section{SUMMARY AND DISCUSSION}

In the present paper we have applied the general EM spontaneous emission theory in Kappa magnetized plasmas [28] for the low frequency fluctuations. Computed for an arbitrary angle of propagation, the emission spectra in this range include the KAW, MSW, KS, IBW, and HOM. The effects of suprathermal populations have been investigated by varying the power index, either for electrons $\left(\kappa_{e}\right)$ or ions $\left(\kappa_{i}\right)$. We have tested the validity of our approach by comparing with the low-frequency fluctuations from PIC simulations, which show a good agreement with theoretical results. 

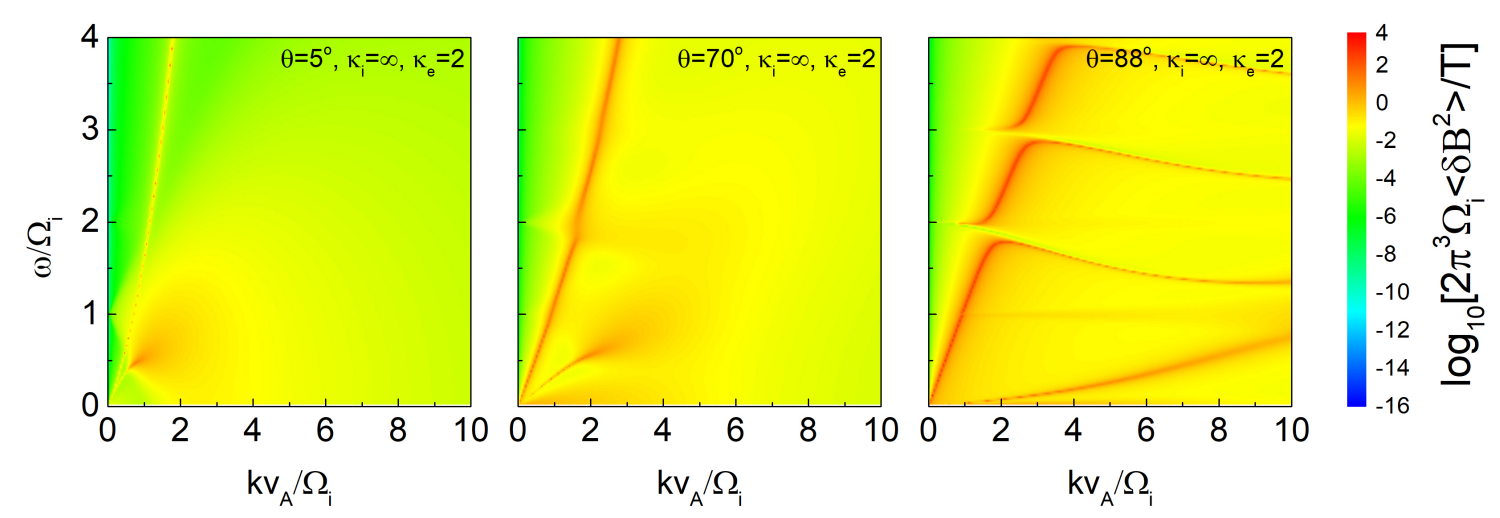

Figure 7. The same as in Figure 5 (for $\kappa_{e}=2$ ) but with relativistic corrections.

The emission spectra is found to be enhanced over the linear eigenmodes of the plasma system, and strengthen as $\kappa$-index decreases and keeping the temperature independent of $\kappa$. This is well explained by the linear resonance between wave fluctuations and suprathermal populations which appear to play an important role, stimulating, in general, the low frequency emissions. The present results are particularly useful for understanding the effects of Kappa distributed ions and electrons on the low frequency emissions.

Although an isotropic temperature was considered, as a specific application, the present work is more generally applicable for anisotropic VDFs considered as quasistable or even unstable against temperature-anisotropy instabilities. Indeed, if we consider the solar wind plasma, the electron and ion VDFs very often deviate from thermal equilibrium, and in the presence of kinetic anisotropies, instabilities must be taken into account, and spontaneous spectra must be considered together with the induced ones. Spontaneous and induced emissions may explain the EM fluctuations inherently present in space plasmas, even in their quasi-stationary states. As expected, the in-situ measurements have confirmed that EM fluctuations are enhanced along the temperature anisotropy thresholds predicted by linear theory [17]. However, for plasma states below these thresholds, the quasi-stable plasma maintain EM fluctuations, at low but still detectable intensities. Recently it was shown that Alfvénic-cyclotron fluctuations propagating along the magnetic field in a plasma composed of thermal and suprathermal protons and electrons may be relevant for understanding the fluctuating EM power observed in the solar wind [5, 6]. Here we have generalized the approach to the full spectrum of wave-vectors. The approach and the results in the present paper might thus contribute to explain the origin of the observed fluctuations.

\section{Acknowledgments}

S.K. and R.S. acknowledge support by the Deutsche Forschungsgesellschaft (Schl 201/321). M.L. and R.S. acknowledge support in the framework of the projects SCHL 
201/35-1 (DFG), and GOA2316N (FWO). R.A.L. acknowledges CONICyT for a BecasChile Postdoctoral fellowship No. 74160076, and CONICyT PIA project ACT1405. P.H.Y. acknowledges NSF grant AGS1550566 to the University of Maryland, the BK21 plus program from the National Research Foundation (NRF), Korea, to Kyung Hee University, and the Science Award Grant from the GFT, Inc., to the University of Maryland.

[1] O. Alexandrova, C. H. K. Chen, L. Sorriso-Valvo, T. S. Horbury, and S. D.Bale, Space Sci. Rev. 178, 101-139 (2009).

[2] R. Bruno, and V. Carbone, Liv. Rev. Solar Phys. 10, 2 (2013).

[3] Gary, S. P., and K. Nishimura J. Geophys. Res. 109, A02109 (2004).

[4] F. Sahraoui, M. L. Goldstein, P. Robert, and Yu.V. Khotyaintsev, Phys. Rev. Lett. 102, 231102 (2009).

[5] R. E. Navarro, P. S. Moya, V. Munoz, J. A. Araneda, A. F.-Viñas, J. A. Valdivia, Phys. Rev. Lett. 112, 245001 (2014).

[6] R. E. Navarro, V. Muñoz, J. A. Araneda, A. F. Viñas, P. S. Moya, and J. A. Valdivia, J. Geophys. Res. 120, 2382 (2015).

[7] Gary, S. P., and C. W. Smith, J. Geophys. Res. 114, A12105 (2009).

[8] Narita,Y.,S.P.Gary,S.Saito,K.H., Glassmeier, and U. Motschmann, Geophys. Res. Lett. 38, L05101 (2011).

[9] G. G. Howes, S. D. Bale, K. G. Klein, C. H. K. Chen, C. S. Salem, and J. M. TenBarge, Astrophys. J. Lett. 753, L19 (2012).

[10] Y. Narita, and E. Marsch, Astrophys. J. 805, 24 (2015).

[11] J. J. Podesta, J. Geophys. Res. 117, A07101 (2012).

[12] J.A. Araneda, H. Astudillo, E. Marsch, Space Sci. Rev. 172, 361 (2012).

[13] M. M. Martinović, A. Zaslavsky, M. Maksimović, N. Meyer-Vernet, S. Šegan, I. Zouganelis, C. Salem, M. Pulupa, and S. D. Bale, J. Geophys. Res. 121, 129 (2016).

[14] M. M. Martinović, A. Zaslavsky, M. Maksimovi ${ }_{3}$ and S. Šegan, J. Geophys. Res. 52, 70 (2017).

[15] I. Zouganelis, J. Geophys. Res. 113, A08111 (2008).

[16] G. Le Chat,K. Issautier, N. Meyer-Vernet, and S. Hoang, Sol. Phys. 271, 141 (2011).

[17] S. D. Bale, J. C. Kasper, G. G. Howes, E. Quataert, C. Salem, and D. Sundkvist, Phys. Rev. Lett. 103, 211101 (2009).

[18] C. S. Wu, G. Q. Zhao, L. Chen, and D. J. Wu, Phys. Plasmas, 20, 082102 (2013).

[19] C. S. Wu, G. Q. Zhao, L. Chen, and D. J. Wu, Phys. Plasmas, 20, 119902 (2013).

[20] P. H. Yoon, R. A. López, S. Vafin, S. Kim, and R. Schlickeiser, Plasma Phys. Control. Fusion 59, $095002(2017)$

[21] R. A. López, A. F. Viñas, J. A. Araneda, and P. H. Yoon, Astrophys. J. 845, 60 (2017)

[22] P. H. Yoon and R. A. López, Phys. Plasmas 24, 022117 (2017).

[23] P. H. Yoon and R. A. López, Phys. Plasmas 24, 049902 (2017).

[24] P. F. Knapp, D. B. Sinars, and K. D. Hahn, Phys. Plasmas 20, 062701 (2013).

[25] S. Gnesin, S. Coda, J. Decker, and Y. Peysson, Rev. Sci. Instrum. 79, 10F504 (2008).

[26] V. Pierrard, and M. Lazar, Solar Phys. 267(1), 153-174 (2010).

[27] M. Maksimovic, V. Pierrard, and P. Riley, Geophys. Res. Lett. 24, 1151-1154 (1997).

[28] S. Kim, R. Schlickeiser, P. H. Yoon, R. A. López, and M. Lazar, Plasma Phys. Control. Fusion 59, 125003 (2017).

[29] R. Gaelzer, and L. F. Ziebell, Phys. Plasmas 23, 022110 (2016).

[30] R. Gaelzer, L. F. Ziebell, and A. R. Meneses, Phys. Plasmas 23, 062108 (2016).

[31] A. F. Viñas, P. S. Moya, R. E. Navarro, and J. A. Araneda, Phys. Plasmas 21, 012902 (2014).

[32] M. J. Michno1, M. Lazar, P. H. Yoon, and R. Schlickeiser, Astrophys. J. 781, 49 (2014).

[33] S. M. Shaaban, M. Lazar, S. Poedts, and A. Elhanbaly, Astrophys. J. 814, 34 (2015).

[34] S. M. Shaaban, M. Lazar, S. Poedts, and A. Elhanbaly, Astrophys. Space Sci. 361, 193 (2016). 
[35] S. M. Shaaban, M. Lazar, S. Poedts, and A. Elhanbaly, Astrophys. Space Sci. 362, 13 (2017).

[36] S. Puri, F. Leuterer, and M. Tutter, J. Plasma Phys. 9, 89 (2013).

[37] F. Nsengiyumva, R. L. Mace, and M. A. Hellberg, Phys. Plasmas 20, 102107 (2013). 